\title{
A Note on a Theorem of A. Connes on Radon-Nikodym Cocycles
}

\author{
By
}

\author{
Tetsuya MASUDA*
}

\begin{abstract}
We give an alternative proof of a theorem of A. Connes that every unitary cocycle (relative to a modular automorphism of a weight $\phi_{0}$ ) is a Radon-Nikodym unitary cocycle $\left(D \phi: D \phi_{0}\right)_{t}$ for some faithful normal semifinite weight $\phi$.
\end{abstract}

\section{§1. Statement of Theorem}

We prove the following theorem of A. Connes ([3], Theorem 1.2. 4) by a method different from his method.

Theorem. Let $\phi_{0}$ be a faithful normal semifinite weight on a von Neumann algebra $M$. Let $\left\{u_{t}\right\}_{t \in R}$ be a strongly continuous one parameter family of unitaries in $M$ satisfying the cocycle condition with respect to the modular automorphism group $\left\{\sigma_{t}^{\phi_{0}}\right\}_{t \in R}$ associated with the weight $\phi_{0}$ i.e.

$$
u_{s+t}=u_{s} \sigma_{s}^{\phi_{0}}\left(u_{t}\right), s, t \in \boldsymbol{R} .
$$

Then there exists a unique faithful normal semifinite weight $\phi$ on $M$ satisfying

$$
\left(D \phi: D \dot{\phi}_{0}\right)_{t}=u_{t}, \quad t \in \boldsymbol{R} .
$$

In the construction of $L_{p}$-spaces ([2], [6]), a generalized version of this theorem for not necessarily unitary $u$ and not necessarily faithful weight $\phi$ plays an important role and is obtained from this theorem, see Appendix of [2] (see also [4]). The construction of $L_{p}$-spaces in [2] and [6] is carried out directly on the relevant von Neumann

Communicated by H. Araki, January 6, 1983.

* Research Institute for Mathematical Sciences, Kyoto University, Kyoto 606, Japan. 
algebra (in contrast to the construction of the same $L_{p}$-spaces using the crossed product of the relevant von Neumann algebra by the modular automorphism group [5]) except for the above theorem, for which the original proof by A. Connes utilizes the tensor product of the relevant von Neumann algebra with a type I factor, a procedure closely related to the crossed product by modular action through Takesaki duality [8]. This has been a motivation for looking for the present alternative proof.

Throughout the paper, we use the standard notion of the TomitaTakesaki theory (for example, see [7]) and relative modular operators (for example, see [1]).

\section{Aknowledgements}

This paper originates from the discussion with Professor M. Takesaki when the author visited U. C. L. A. in August, 1980, after Kingston conference. Further, the most important idea of the proof is due to Professor M. Takesaki to whom the author would like to express his hearty thanks. The author also expresses his hearty thanks to Professor H. Araki for valuable discussions and comments.

\section{§2. Proof of the Theorem}

We prove the theorem in several steps. We introduce a $\sigma$-weakly continuous one parameter group of isometric linear transformations $\left\{\alpha_{t}\right\}_{t \in \boldsymbol{R}}$ and automorphisms $\left\{\beta_{t}\right\}_{t \in \boldsymbol{R}}$ as follows:

$$
\begin{gathered}
\alpha_{t}(x)=u_{t} \sigma_{t}^{\phi_{0}}(x), \\
\beta_{t}(x)=u_{t} \sigma_{t}^{\phi_{0}}(x) u_{t}^{*}, x \in M, t \in \boldsymbol{R} .
\end{gathered}
$$

By the equality $\alpha_{t}(x)^{*} \alpha_{t}(x)=\sigma_{t}^{\phi_{0}}\left(x^{*} x\right), x \in M, t \in \boldsymbol{R}, \alpha_{t}$ leaves $N_{\phi_{0}}$ invariant.

Lemma 2.1. There exists a positive self-adjoint operator $T$ on $H_{\phi_{0}}$ satisfying

$$
\begin{gathered}
u_{t}=T^{i t} \Delta_{\phi_{0}}^{-i t}, \\
T^{i t} \eta_{\phi_{0}}(x)=\eta_{\phi_{0}}\left(\alpha_{t}(x)\right), x \in N_{\phi_{0}}, t \in \boldsymbol{R} .
\end{gathered}
$$


Proof. Let $T(t)$ be a strongly continuous one parameter family of unitaries on $H_{\phi_{0}}$ defined by

$$
T(t)=u_{t} \Delta_{\phi_{0}}^{i t}, t \in \boldsymbol{R} .
$$

By the cocycle condition (1.1), $\{T(t)\}_{t \in \boldsymbol{R}}$ is a group. Hence there exists a self-adjoint operator $T$ such that $T(t)=T^{i t}$ by Stone's theorem and (2.3) follows. The formula (2.4) follows from (2.1) and (2.5).

Q. E. D.

We denote by $\widetilde{N}_{\phi_{0}}$ the set of all entire analytic elements of $N_{\phi_{0}}$ with respect to $\left\{\alpha_{t}\right\}_{t \in \boldsymbol{R}}$. We also denote by $M_{\phi_{0}}$ the set of all entire analytic elements of $N_{\phi_{0}}^{*} \cap N_{\phi_{0}}$ with respect to the modular action $\left\{\sigma_{t}^{\phi_{0}}\right\}_{t \in \boldsymbol{R}}$. We denote by $\tilde{N}_{\phi_{0}} \tilde{N}_{\phi_{0}}^{*}$ the set of all $\boldsymbol{C}$-linear combinations of the elements $x y^{*}, x, y \in \widetilde{N}_{\phi_{0}}$. By (2.1),

$$
\alpha_{t}(x)^{*} \alpha_{t}(y)=\sigma_{t}^{\phi_{0}}\left(x^{*} y\right)
$$

and hence, $\tilde{N}_{\phi_{0}}^{*} \tilde{N}_{\phi_{0}} \subset M_{\phi_{0}}$.

Now, we define a mapping $\eta: \widetilde{N}_{\phi_{0}} \tilde{N}_{\phi_{0}}^{*} \rightarrow H_{\phi_{0}}$ by

$$
\eta\left(\sum_{k=1}^{n} x_{k} y_{k}^{*}\right)=\sum_{k=1}^{n} x_{k} J_{\phi_{0}} T^{1 / 2} \eta_{\phi_{0}}\left(y_{k}\right),
$$

where $x_{k}, y_{k} \in \tilde{N}_{\phi_{0}}, k=1, \cdots, n$.

Lemma 2.2. The formula (2.7) gives a well-defined injective linear mapping $\eta$ from $\tilde{N}_{\phi_{0}} \widetilde{N}_{\phi_{0}}^{*}$ to $H_{\phi_{0}}$ with a dense range.

Proof. If we show that $\eta$ is well-defined, then the linearity of $\eta$ follows. Suppose $\sum_{k=1}^{n} x_{k} y_{k}^{*}=0, x_{k}, y_{k} \in \tilde{N}_{\phi_{0}}, k=1, \cdots, n$. Then

$$
\begin{aligned}
& \text { II } \sum_{k=1}^{n} x_{k} J_{\phi_{0}} T^{1 / 2} \eta_{\phi_{0}}\left(y_{k}\right) \|^{2} \\
& =\sum_{k=1}^{n} \sum_{l=1}^{n}\left(x_{k} J_{\phi_{0}} T^{1 / 2} \eta_{\phi_{0}}\left(y_{k}\right), x_{l} J_{\phi_{0}} T^{1 / 2} \eta_{\phi_{0}}\left(y_{l}\right)\right) \\
& =\sum_{k=1}^{n} \sum_{l=1}^{n}\left(J_{\phi_{0}} x_{k}^{*} x_{l} J_{\phi_{0}} \eta_{\phi_{0}}\left(\alpha_{-i / 2}\left(y_{l}\right)\right), T^{1 / 2} \eta_{\phi_{0}}\left(y_{k}\right)\right) \\
& =\sum_{k=1}^{n} \sum_{l=1}^{n}\left(\alpha_{-i / 2}\left(y_{l}\right) J_{\phi_{0}} \eta_{\phi_{0}}\left(x_{k}^{*} x_{l}\right), T^{1 / 2} \eta_{\phi_{0}}\left(y_{k}\right)\right) \\
& =\sum_{k=1}^{n} \sum_{l=1}^{n}\left(\alpha_{-i / 2}\left(y_{l}\right) \eta_{\phi_{0}}\left(\sigma_{-i / 2}^{\phi_{0}}\left(x_{l}^{*} x_{k}\right)\right), T^{1 / 2} \eta_{\phi_{0}}\left(y_{k}\right)\right) \\
& =\sum_{k=1}^{n}\left(\eta_{\phi_{0}}\left(\alpha_{-i / 2}\left\{\left[\sum_{l=1}^{n} x_{l} y_{l}^{*}\right]^{*} x_{k}\right\}\right), T^{1 / 2} \eta_{\phi_{0}}\left(y_{k}\right)\right) \\
& =0,
\end{aligned}
$$


where we used $J_{\phi_{0}} x J_{\phi_{0}} \eta_{\phi_{0}}(y)=y J_{\phi_{0}} \eta_{\phi_{0}}(x), x, y \in N_{\phi_{0}}$ for the third equality, $\tilde{N}_{\phi_{0}}^{*} \tilde{N}_{\phi_{0}} \subset M_{\phi_{0}}$ for the fourth equality and the analytic continuation of $\alpha_{t}(x) \sigma_{t}^{\phi_{0}}(y)=\alpha_{t}(x y), x \in \tilde{N}_{\phi_{0}}, y \in M_{\phi_{0}}(t \longmapsto-i / 2)$ for the fifth equality. The density of the range follows from $T^{1 / 2} \eta_{\phi_{0}}(y)=$ $\eta_{\phi_{0}}\left(\alpha_{-i / 2}(y)\right), \alpha_{-i / 2}\left(\tilde{N}_{\phi_{0}}\right)=\tilde{N}_{\phi_{0}}$, the density of $\eta_{\phi_{0}}\left(\tilde{N}_{\phi_{0}}\right)$ in $H_{\phi_{0}}, J_{\phi_{0}}^{2}=1$ and the strong density of $\tilde{N}_{\phi_{0}}$ in $M$ (so that we may take $n=1$ and approximate 1 by $x_{1}$ ).

Next, we show that $\eta$ is injective. Suppose $\eta\left(\sum_{k=1}^{n} x_{k} y_{k}^{*}\right)=0, x_{k}, y_{k}$ $\in \tilde{N}_{\phi_{0}}$. By the same calculation as (2.8), we obtain $0=\left(\eta\left(\sum_{k=1}^{n} x_{k} y_{k}^{*}\right)\right.$, $\left.\eta\left(x y^{*}\right)\right)=\left(T^{1 / 2} \eta_{\phi_{0}}\left(\left[\sum_{k=1}^{n} x_{k} y_{k}^{*}\right]^{*} x\right), T^{1 / 2} \eta_{\phi_{0}}(y)\right)$ for any $x, y \in \tilde{N}_{\phi_{0}}^{k=1}$ The density of $T^{1 / 2} \eta_{\phi_{0}}\left(\tilde{N}_{\phi_{0}}^{k=1}\right)$ implies $T^{1 / 2} \eta_{\phi_{0}}\left(\left[\sum_{k=1}^{n} x_{k} y_{k}^{*}\right]^{*} x\right)=0$. Because $T$ is nonsingular and $\phi_{0}$ is faithful, $\left[\sum_{k=1}^{n} x_{k} y_{k}^{*}\right]^{*} x=0$. By taking limit $x \rightarrow 1$, we obtain $\sum_{k=1}^{n} x_{k} y_{k}^{*}=0$. Thus the injectivity of $\eta$ follows. Q.E.D.

Now, we set $\mathfrak{A}_{0}=\eta\left(\tilde{N}_{\phi_{0}} \tilde{N}_{\phi_{0}}^{*}\right)$ with the following algebraic operations $\eta(x) \eta(y)=\eta(x y), \eta(x)^{\sharp}=\eta\left(x^{*}\right), \Delta(z) \eta(x)=\eta\left(\beta_{-i z}(x)\right), z \in C$ (note that by (2.1) and (2.2), $\alpha_{t}(x) \alpha_{t}(y)^{*}=\beta_{t}\left(x y^{*}\right)$ and hence the elements of $\widetilde{N}_{\phi_{0}} \widetilde{N}_{\phi_{0}}^{*}$ are entire alalytic with respect to the automorphism group $\left\{\beta_{t}\right\}_{t \in R}$ and $\tilde{N}_{\phi_{0}} \tilde{N}_{\phi_{0}}^{*}$ is $\beta$-invariant due to the $\alpha$-invariance of $\tilde{N}_{\phi_{0}}$ ).

\section{Lemma 2.3.}

(1) The mapping $z \in C \longmapsto \Delta(z) \xi$ is analytic for $\xi \in \mathfrak{A}_{0}$.

$$
\begin{aligned}
& \left(\Delta(z) \xi_{1}, \xi_{2}\right)=\left(\tilde{\xi}_{1}, \Delta(\bar{z}) \xi_{2}\right), \xi_{1}, \xi_{2} \in \mathfrak{A}_{0}, z \in \boldsymbol{C} . \\
& \left(\Delta(1) \xi_{1}, \xi_{2}\right)=\left(\xi_{2}^{\sharp}, \xi_{1}^{\sharp}\right), \xi_{1}, \xi_{2} \in \mathfrak{A}_{0} .
\end{aligned}
$$

Proof. (1) Let $x, y \in \tilde{N}_{\phi_{0}}$. Then

$$
\begin{aligned}
& \Delta(z) \eta\left(x y^{*}\right)=\eta\left(\beta_{-i z}\left(x y^{*}\right)\right)=\eta\left(\alpha_{-i z}(x) \alpha_{i \bar{z}}(y)^{*}\right) \\
& =\alpha_{-i z}(x) J_{\phi_{0}} T^{(1 / 2)-\bar{z}} \eta_{\phi_{0}}(y) .
\end{aligned}
$$

Hence we obtain the analyticity of the mapping $z \longmapsto \Delta(z) \xi, \xi \in \mathfrak{A}_{0}$.

(2) First, we show the unitarity of $\Delta(i t), t \in \boldsymbol{R}$. Let $x_{k}, y_{k} \in \tilde{N}_{\phi_{0}}$, $k=1$, 2. Then

$$
\begin{aligned}
& \left(\Delta(i t) \eta\left(x_{1} y_{1}^{*}\right), \Delta(i t) \eta\left(x_{2} y_{2}^{*}\right)\right) \\
& =\left(\eta\left(\alpha_{t}\left(x_{1}\right) \alpha_{t}\left(y_{1}\right)^{*}\right), \eta\left(\alpha_{t}\left(x_{2}\right) \alpha_{t}\left(y_{2}\right)^{*}\right)\right) \\
& =\left(\alpha_{t}\left(x_{1}\right) J_{\phi_{0}} T^{(1 / 2)+i t} \eta_{\phi_{0}}\left(y_{1}\right), \alpha_{t}\left(x_{2}\right) J_{\phi_{0}} T^{(1 / 2)+i t} \eta_{\phi_{0}}\left(y_{2}\right)\right)
\end{aligned}
$$




$$
\begin{aligned}
& =\left(x_{1} \Delta_{\phi_{0}}^{-i t} J_{\phi_{0}} T^{i t} T^{1 / 2} \eta_{\phi_{0}}\left(y_{1}\right), x_{2} \Delta_{\phi_{0}}^{-i t} J_{\phi_{0}} T^{i t} T^{1 / 2} \eta_{\phi_{0}}\left(y_{2}\right)\right) \\
& =\left(x_{1} J_{\phi_{0}} u_{-t}^{*} T^{1 / 2} \eta_{\phi_{0}}\left(y_{1}\right), x_{2} J_{\phi_{0}} u_{-t}^{*} T^{1 / 2} \eta_{\phi_{0}}\left(y_{2}\right)\right) \\
& =\left(J_{\phi_{0}} u_{-t}^{*} J_{\phi_{0}} x_{1} J_{\phi_{0}} T^{1 / 2} \eta_{\phi_{0}}\left(y_{1}\right), J_{\phi_{0}} u_{-t}^{*} J_{\phi_{0}} x_{2} J_{\phi_{0}} T^{1 / 2} \eta_{\phi_{0}}\left(y_{2}\right)\right) \\
& =\left(x_{1} J_{\phi_{0}} T^{1 / 2} \eta_{\phi_{0}}\left(y_{1}\right), x_{2} J_{\phi_{0}} T^{1 / 2} \eta_{\phi_{0}}\left(y_{2}\right)\right) \\
& =\left(\eta\left(x_{1} y_{1}^{*}\right), \eta\left(x_{2} y_{2}^{*}\right)\right) .
\end{aligned}
$$

By the density of the range and domain of $\Delta(z), \Delta(i t)$ is unitary. Hence, we obtain $\left(\Delta(i t) \xi_{1}, \xi_{2}\right)=\left(\xi_{1}, \Delta(-i t) \xi_{2}\right), \xi_{1}, \xi_{2} \in \mathfrak{U}_{0}$ due to the group property of $\{\Delta(z)\}_{z \in c}$. By the analyticity of two functions $z \longmapsto\left(\Delta(z) \xi_{1}, \xi_{2}\right)$ and $z \longmapsto\left(\xi_{1}, \Delta(\bar{z}) \xi_{2}\right)$, (see (1)), we obtain (2),

(3) Let $x_{k}, y_{k} \in \tilde{N}_{\phi_{0}}, k=1,2$. Then

$$
\begin{aligned}
& \left(\Delta(1) \eta\left(x_{1} y_{1}^{*}\right), \eta\left(x_{2} y_{2}^{*}\right)\right) \\
& =\left(\Delta(1 / 2) \Delta(1 / 2) \eta\left(x_{1} y_{1}^{*}\right), \eta\left(x_{2} y_{2}^{*}\right)\right) \\
& =\left(\Delta(1 / 2) \eta\left(x_{1} y_{1}^{*}\right), \Delta(1 / 2) \eta\left(x_{2} y_{2}^{*}\right)\right) \\
& =\left(\alpha_{-i / 2}\left(x_{1}\right) J_{\phi_{0}} \eta_{\phi_{0}}\left(y_{1}\right), \alpha_{-i / 2}\left(x_{2}\right) J_{\phi_{0}} \eta_{\phi_{0}}\left(y_{2}\right)\right) \\
& =\left(J_{\phi_{0}} y_{1} J_{\phi_{0}} \eta_{\phi_{0}}\left(\alpha_{-i / 2}\left(x_{1}\right)\right), J_{\phi_{0}} y_{2} J_{\phi_{0}} \eta_{\phi_{0}}\left(\alpha_{-i / 2}\left(x_{2}\right)\right)\right) \\
& =\left(y_{2} J_{\phi_{0}} T^{1 / 2} \eta_{\phi_{0}}\left(x_{2}\right), y_{1} J_{\phi_{0}} T^{1 / 2} \eta_{\phi_{0}}\left(x_{1}\right)\right) \\
& =\left(\eta\left(y_{2} x_{2}^{*}\right), \eta\left(y_{1} x_{1}^{*}\right)\right),
\end{aligned}
$$

where we used (2) with $z=1 / 2$ for the second equality, and (2.9) for the third equality. Hence we obtain (3).

Q. E. D.

Lemma 2.4. Equipped with the above structure, $\mathfrak{A}_{0}$ is a left Hilbert algebra with its left von Neumann algebra $M$.

Proof. It is easy to see that $\eta(x)=0$ implies $\eta(x y)=0, \eta(y x)=0$ and $\eta\left(x^{*}\right)=0$ due to the injectivity of $\eta$ in Lemma 2.2, $(\xi \eta, \zeta)=$ $\left(\eta, \xi^{\sharp} \zeta\right)$ for $\xi, \eta, \zeta \in \mathfrak{A}_{0}$, the boundedness of the left multiplication $\mathfrak{U}_{0} \ni \eta \longmapsto \xi \eta \in \mathfrak{U}_{0}, \xi \in \mathfrak{U}_{0}$, the density of $\mathfrak{U}_{0} \mathfrak{U}_{0}$ in $\mathfrak{A}_{0}$ (which follows from the $\sigma$-weak density of $\tilde{N}_{\phi_{0}}$ in $M$ ). Now, we prove the preclosedness of $\xi \longmapsto \xi^{\sharp}$. We define $\xi \longmapsto \xi^{b}$ by $\eta(x)^{\prime}=\eta\left(\beta_{-i}\left(x^{*}\right)\right)$. Let $x_{k}, y_{k}$ $\in \tilde{N}_{\phi_{0}}, k=1,2$. Then,

$$
\begin{aligned}
& \left(\eta\left(x_{1} y_{1}^{*}\right)^{b}, \eta\left(x_{2} y_{2}^{*}\right)\right)=\left(\Delta(1) \eta\left(y_{1} x_{1}^{*}\right), \eta\left(x_{2} y_{2}^{*}\right)\right) \\
= & \left(\eta\left(y_{2} x_{2}^{*}\right), \eta\left(x_{1} y_{1}^{*}\right)\right)=\left(\eta\left(x_{2} y_{2}^{*}\right)^{\#}, \eta\left(x_{1} y_{1}^{*}\right)\right),
\end{aligned}
$$

where we used Lemma 2.3 (3) for the second equality. This shows the preclosedness of $\xi \longmapsto \xi^{\#}$.

By the $\sigma$-weak density of $\tilde{N}_{\phi_{0}}$ in $M, \mathfrak{A}_{0}$ is a $\sigma$-weakly dense 
*-subalgebra of $M$ through the left multiplication, hence the corresponding left von Neumann algebra is $M$.

Q. E. D.

Now, we obtain a faithful normal semifinite weight $\phi$ on $M$ given by the achieved left Hilbert algebra $\tilde{\mathfrak{A}}_{0}$ given by the left Hilbert algebra $\mathfrak{A}_{0}$ obtained above. By the construction, $\left\{\beta_{t}\right\}_{t \in \boldsymbol{R}}$ is the modular automorphism group of the weight $\phi$.

Proof of the Theorem. By the construction of the mapping $\eta$ given by (2.7),

$$
\eta_{\phi}\left(x y^{*}\right)=I\left(\phi, \phi_{0}\right) x J_{\phi_{0}} T^{1 / 2} \eta_{\phi_{0}}(y),
$$

where $x, y \in \tilde{N}_{\phi_{0}}$ and $I\left(\phi, \phi_{0}\right)$ is the unitary operator identifying the standard representation spaces $H_{\phi_{0}} \longrightarrow H_{\phi}$. Hence we obtain,

$$
\eta_{\phi}\left(x y^{*}\right)=x J_{\phi, \phi_{0}} T^{1 / 2} \eta_{\phi_{0}}(y), \quad x, y \in \tilde{N}_{\phi_{0}} \text {. }
$$

It follows that $\tilde{N}_{\phi_{0}}^{*} \subset N_{\phi}$ and

$$
\Delta_{\phi, \phi_{0}}^{1 / 2} \eta_{\phi_{0}}(y)=T^{1 / 2} \eta_{\phi_{0}}(y), y \in \tilde{N}_{\phi_{0}} .
$$

By construction, $\eta_{\phi_{0}}\left(\tilde{N}_{\phi_{0}}\right)$ is a core for $T^{1 / 2}$, we obtain $\Delta_{\phi, \phi_{0}}^{1 / 2} \supset T^{1 / 2}$. Since both sides are self-adjoint, we obtain $\Delta_{\phi, \phi_{0}}^{1 / 2}=T^{1 / 2}$ and hence we obtain $u_{t}=T^{i t} \Delta_{\phi_{0}}^{-i t}=\Delta_{\phi, \phi_{0}}^{i t} \Delta_{\phi_{0}}^{-i t}=\left(D \phi: D \phi_{0}\right)_{t}$. Q. E. D.

\section{References}

[1] Araki, H., Introduction to relative Hamiltonian and relative entropy, Lecture Notes at Marseille, 1975.

[2] Araki, H. and Masuda, T., Positive cones and $L_{p}$-spaces for von Neumann algebra, Publ. RIMS Kyoto Univ., 18 (1982), 759-831.

[3] Connes, A., Une classification des facteurs de type III, Ann. Scie. École Norm. Sup. 4 ème sér. 6 (1973), 133-252.

[4] Connes, A. and Takesaki, M., The flow of weights on factors of type III, Tohoku Math. J. 29 (1977), 473-575.

[5] Haagerup, U., $L^{p}$-spaces associated with an arbitrary von Neumann algebra, Colloques Internationaux C. N. R.S. $\mathbf{N}^{\circ} \mathbf{2 7 4}, 175-184$.

[6] Masuda, T., $L_{p}$-spaces for von Neumann algebra with reference to a faithful normal semifinite weight, Publ. RIMS Kyoto Univ. 19 (1983), 673-727.

[7] Takesaki, M., Tomita's theory of modular Hilbert algebras and its applications, Lecture Notes in Math., 128 (1970), Springer.

[8] Duality for crossed products and the structure of von Neumann algebras of type III, Acta Math., 131 (1973), 249-308. 